\title{
Random Fuzzy Differential Equations with Impulses
}

\author{
Ho Vu \\ Faculty of Mathematical Economics, Banking University of Ho Chi Minh City, Ho Chi Minh City, Vietnam \\ Correspondence should be addressed to Ho Vu; vuh@buh.edu.vn
}

Received 9 April 2017; Accepted 27 April 2017; Published 19 June 2017

Academic Editor: Omar Abu Arqub

Copyright (C) 2017 Ho Vu. This is an open access article distributed under the Creative Commons Attribution License, which permits unrestricted use, distribution, and reproduction in any medium, provided the original work is properly cited.

We consider the random fuzzy differential equations (RFDEs) with impulses. Using Picard method of successive approximations, we shall prove the existence and uniqueness of solutions to RFDEs with impulses under suitable conditions. Some of the properties of solution of RFDEs with impulses are studied. Finally, an example is presented to illustrate the results.

\section{Introduction}

Impulsive differential equations (IDEs) are a new branch of differential equations. IDEs can find numerous applications in different branches of optimal control, electronics, economics, physics, chemistry, and biological sciences. We refer to [1-4] and the references therein. As we know, the real systems are often faced with two kinds of uncertainties (fuzziness and randomness). Therefore, this topic has extensively been studied by mathematicians in recent years. Investigations of dynamic systems with fuzziness have been developed in connection with fuzzy differential equations (FDEs). Evidence of FDEs for such areas as control theory, differential inclusions, and fuzzy differential equations can be found in the papers of [5-8], the books and monographs [9], and references therein. In [10], Lakshmikantham and McRae combined the theories of impulsive differential equations and fuzzy differential equations. There are a few papers on the latter topic; see [10-12].

Moreover, the class of random fuzzy differential equations (RFDEs) could be applicable in the investigation of numerous engineering and economics problems where the phenomena are simultaneously subjected to two kinds of uncertainties, that is, fuzziness and randomness, simultaneously (see, e.g., Malinowski [13-16], Feng [17, 18], and Fei [19, 20]). Feng [17] introduced the concepts by the mean-square derivative and mean-square integral of second-order fuzzy stochastic processes. Using the results, the author [18] investigated the properties of solutions of the fuzzy stochastic differential systems, including the existence and uniqueness of solution, the dependence of the solution of the initial condition, and the continuity and the boundedness of solution of systems when there are perturbations of the coefficients and the initial conditions. In $[19,20]$, Fei proved the existence and uniqueness of solution of fuzzy random differential equation (FRDE). The author also discussed the dependence of solution to FRDE on initial values. Finally, the nonconfluence property of the solution for FRDE is studied.

In [13], Malinowski considered the following random fuzzy differential equations:

$$
\begin{aligned}
D_{H} x(t, \omega) & \stackrel{\left[t_{0}, t_{0}+p\right], \mathbb{P} .1}{=} f(t, x(t, \omega)), \\
x\left(t_{0}, \omega\right) & \stackrel{\mathbb{P} .1}{=} x_{0}(\omega) \in E^{d},
\end{aligned}
$$

where $f: \Omega \times\left[t_{0}, t_{0}+p\right] \times E^{d} \rightarrow E^{d}$ and the symbol $D_{H}$ denotes the fuzzy Hukuhara derivative. The author proved the existence and uniqueness of the solution for RFDEs under Lipschitz condition. Malinowski $[14,15]$ studied two kinds of solutions to the RFDEs with two kinds of fuzzy derivatives. For both cases the author established the existence and uniqueness of local solutions to RFDEs. In addition, the author also presented some examples being simple illustrations of the theory of RFDEs.

Inspired and motivated by Fei [19], Feng [18], Malinowski [14], and other authors as in $[3,10,21]$, in this paper, we consider the RFDEs with impulses under Hukuhara derivative. The paper is organized as follows: in Section 2, we summarize 
some preliminary facts and properties of the fuzzy set space, fuzzy differentiation, and integration. We also recall the notions of fuzzy random variable and fuzzy stochastic process. In Section 3, we discuss the RFDEs with impulses. Under suitable conditions, we prove the existence and uniqueness of solutions to RFDEs with impulses. In Section 4, we give some examples to illustrate these results.

\section{Preliminaries}

In this section, we give some definitions and properties and introduce the necessary notation which will be used throughout the paper. We denote $E^{d}=\left\{u: \mathbb{R}^{d} \rightarrow[0,1] \mid u\right.$ satisfies (i)-(iv) stated below\}, where

(i) $u$ is normal; that is, there exists an $x_{0} \in \mathbb{R}^{d}$ such that $u\left(x_{0}\right)=1$;

(ii) $u$ is fuzzy convex; that is, for $0 \leq \lambda \leq 1, u\left(\lambda x_{1}+(1-\right.$ $\left.\lambda) x_{2}\right) \geq \min \left\{u\left(x_{1}\right), u\left(x_{2}\right)\right\}$, for any $x_{1}, x_{2} \in \mathbb{R}^{d}$;

(iii) $u$ is upper semicontinuous;

(iv) $c l\left\{x \in \mathbb{R}^{d}: u(z)>0\right\}$ is compact set.

Then $E^{d}$ is called the space of fuzzy numbers.

For $0<\alpha \leq 1$, we denote $[u]^{\alpha}=\left\{x \in \mathbb{R}^{d} \mid u(x) \geq \alpha\right\}$ and $[u]^{0}=\operatorname{cl}\{x \in \mathbb{R} \mid u(x)>0\}$. For $d=1$ and from conditions (i)-(iv), we infer that the $\alpha$-level cut of $u$, denoted by $[u]^{\alpha}$, is a bounded closed interval for any $\alpha \in[0,1]$ and $u \in E^{d}$, and $[u]^{\alpha}=\left[u_{\alpha}^{l}, u_{\alpha}^{r}\right]$, where $u_{\alpha}^{l}$ and $u_{\alpha}^{r}$ are the lower and upper branches of $u$.

For $u, v \in E^{d}$, the Hausdorff distance between $u$ and $v$ is defined by

$$
d_{\infty}(x, y)=\sup _{\alpha \in[0,1]} \max \left\{d_{H}\left([u]^{\alpha}\right), d_{H}\left([v]^{\alpha}\right)\right\}
$$

and $\left(E^{d}, d_{\infty}\right)$ is a complete metric space.

If we define $D: E^{d} \times E^{d} \rightarrow \mathbb{R}_{+}$by the expression

$$
D(u, v)=\sup _{t \in[a, b]} d_{\infty}(u(t), v(t))
$$

then it is well-known that $D$ is metric in $E^{d}$ and $\left(E^{d}, D\right)$ is also a complete metric space.

Some properties are well-known for the metric Hausdorff $D$ defined on $E^{d}$ as follows:

$$
\begin{aligned}
& D(u+w, v+w)=D(u, v), \\
& D(\lambda u, \lambda v)=\lambda D(u, v), \\
& D(u, v) \leq D(u, w)+D(v, w),
\end{aligned}
$$

for every $u, v, w \in E^{d}$ and $\lambda \in \mathbb{R}_{+}$.

Definition 1 (see [22]). Let $u, v \in E^{d}$. If there exists $w \in E^{d}$ such that $u=v+w$, then $w$ is called the Hukuhara difference of $u, v$ and it is denoted by $u \ominus v$.
Definition 2 (see [22]). Let $f:(a, b) \rightarrow E^{d}$ and $t \in(a, b)$. We say that $f$ is differentiable at $t$ if there exists an element $D_{H} f(t) \in E^{d}$ such that the limits

$$
\lim _{h \rightarrow 0^{+}} \frac{f(t+h) \ominus f(t)}{h}=\lim _{h \rightarrow 0^{+}} \frac{f(t) \ominus f(t-h)}{h}
$$

exist and are equal to $D_{H} f(t)$.

Definition 3 (see [22]). Let $f:(a, b) \rightarrow E^{d}$. The integral of $f$ on $(a, b)$, denoted by $\int_{a}^{b} f(t) d t$, is defined levelwise by the equation

$$
\begin{aligned}
& {\left[\int_{a}^{b} f(t) d t\right]^{\alpha}=\int_{a}^{b}[f(t)]^{\alpha} d t} \\
& \quad=\left\{\int_{a}^{b} \widetilde{f}(t) d t \mid \tilde{f}:(a, b)\right. \\
& \left.\quad \longrightarrow \mathbb{R} \text { is a measurable selection for }[f(\cdot)]^{\alpha}\right\},
\end{aligned}
$$

for all $\alpha \in[0,1]$.

Definition 4 (see [22]). A fuzzy mapping $f:(a, b) \rightarrow E^{d}$ is integrable if $f$ is integrable bounded and strongly measurable.

The following are some properties of integrability of fuzzy mapping (see [22]):

(a) If $f:(a, b) \rightarrow E^{d}$ is continuous then it is integrable.

(b) If $f:(a, b) \rightarrow E^{d}$ is integrable and $c \in(a, b)$ then $\int_{a}^{b} f(s) d s=\int_{a}^{c} f(s) d s+\int_{c}^{b} f(s) d s$.

(c) Let $f, g:(a, b) \rightarrow E^{d}$ be integrable and $\lambda>0$. Then

$$
\begin{aligned}
& \text { (i) } \int_{a}^{b}(f(s)+g(s)) d s=\int_{a}^{b} f(s) d s+\int_{a}^{b} g(s) d s \text {, } \\
& \text { (ii) } \int_{a}^{b} \lambda f(s) d s=\lambda \int_{a}^{b} f(s) d s \text {, } \\
& \text { (iii) } D(f, g) \text { is integrable and } \\
& D\left(\int_{a}^{b} f(s) d s, \int_{a}^{b} g(s) d s\right) \leq \int_{a}^{b} D(f(s), g(s)) d s .
\end{aligned}
$$

Let $(\Omega, \mathscr{F}, \mathbb{P})$ be a complete probability space. A function $x: \Omega \rightarrow E^{d}$ is called a fuzzy random variable, if the setvalued mapping $[x(\cdot)]^{\alpha}: \Omega \rightarrow \mathscr{K}_{c}\left(\mathbb{R}^{d}\right)$ is a measurable multifunction for all $\alpha \in[0,1]$; that is,

$$
\left\{\omega \in \Omega \mid[x(\omega)]^{\alpha} \cap B \neq \emptyset\right\} \in \mathscr{F}
$$

for every closed set $B \subset \mathbb{R}^{d}$.

Definition 5 (see [13]). A mapping $x:[a, b] \times \Omega \rightarrow E^{d}$ is said to be a fuzzy stochastic process if $x(\cdot, \omega)$ is a fuzzy-set-valued function with any fixed $\omega \in \Omega$ and $x(t, \cdot)$ is a fuzzy random variable for any fixed $t \in[a, b]$.

Definition 6 (see [13]). A fuzzy stochastic process $x:[a, b] \times$ $\Omega \rightarrow E^{d}$ is called continuous if there exists $\Omega_{0} \subset \Omega$ with $\mathbb{P}\left(\Omega_{0}\right)=1$ and such that, for every $\omega \in \Omega_{0}$, the trajectory $x(\cdot, \omega)$ is a continuous function on $[a, b]$ with respect to the metric $D$. 
For convenience, from now on, we shall write $x(\omega) \stackrel{\mathbb{P} .1}{=}$ $y(\omega)$ to replace $\mathbb{P}(\{\omega \mid x(\omega)=y(\omega)\})=1$ for short, where $x$, $y$ are random elements, and similarly for inequalities. Also we shall write $x(t, \omega) \stackrel{[a, b], \mathbb{P} .1}{=} y(t, \omega)$ to replace $\mathbb{P}(\{\omega \mid x(t, \omega)=$ $y(t, \omega)\}, \forall t \in[a, b])=1$ for short, where $x, y$ are some stochastic processes, and similarly for inequalities.

\section{Existence and Uniqueness for RFDEs with Impulses}

In this section, we consider the following random fuzzy differential equation with impulses:

$$
\begin{aligned}
& D_{H} x(t, \omega) \stackrel{\mathbb{P} .1}{=} f(t, x(t, \omega), \omega), \\
& \qquad t \in J:=\left[t_{0}, t_{0}+p\right], t \neq t_{k}, \\
& x\left(t_{k}^{+}, \omega\right) \stackrel{\mathbb{P} .1}{=} I_{k}\left(x\left(t_{k}, \omega\right), \omega\right), \quad k=\overline{1, m}, t=t_{k}, \\
& x\left(t_{0}^{+}, \omega\right) \stackrel{\mathbb{P} .1}{=} x_{0}(\omega) \in E^{d},
\end{aligned}
$$

where $f: J \times E^{d} \times \Omega \rightarrow E^{d}, I_{k}: E^{d} \times \Omega \rightarrow E^{d}$ is continuous with $\mathbb{P} .1$, and $t_{k}, k=\overline{1, m}$, are points of impulses such that $t_{0} \leq \cdots<t_{k}<t_{k+1} \leq t_{0}+p$ and $x_{0}: \Omega \rightarrow E^{d}$ is fuzzy random variable.

Lemma 7. Let $x: J \times \Omega \rightarrow E^{d}$ be a fuzzy stochastic process. Then $x$ is the solution of problem (8) if and only if $x$ is a continuous fuzzy stochastic process and satisfy the following random impulsive fuzzy integral equation:

$$
\begin{aligned}
x(t, \omega)= & x_{0}(\omega)+\int_{t_{0}}^{t} f(s, x(s, \omega), \omega) d s \\
& +\sum_{i=1}^{k} I_{i}\left(x\left(t_{i}, \omega\right), \omega\right) .
\end{aligned}
$$

Proof. We divide the proof into two steps.

Step 1. If $x(t)$ satisfies problem (8), then it will be expressed as (9). Indeed, for every $t \in\left[t_{0}, t_{1}\right)$ we have

$$
D_{H} x(t, \omega) \stackrel{\mathbb{P} .1}{=} f(t, x(t, \omega), \omega) .
$$

By Lemma 3.1 in [13], we obtain

$$
x\left(t_{1}, \omega\right) \stackrel{\left[t_{0}, t_{1}\right), \mathbb{P} .1}{=} x_{0}(\omega)+\int_{t_{0}}^{t_{1}} f(s, x(s, \omega), \omega) d s .
$$

If $t \in\left[t_{1}, t_{2}\right)$ and by Lemma 3.1 in [13], we have

$$
\begin{aligned}
x(t, \omega) \stackrel{\mathbb{P} .1}{=} & x\left(t_{1}^{+}, \omega\right)+\int_{t_{1}}^{t} f(s, x(s, \omega), \omega) d s \\
\stackrel{\mathbb{P} .1}{=} & I_{1}\left(x_{1}(t, \omega), \omega\right)+\int_{t_{1}}^{t} f(s, x(s, \omega), \omega) d s \\
\stackrel{\mathbb{P} .1}{=} & I_{1}\left(x_{1}(t, \omega), \omega\right)+x_{0}(\omega) \\
& +\int_{t_{0}}^{t_{1}} f(s, x(s, \omega), \omega) d s \\
& +\int_{t_{1}}^{t} f(s, x(s, \omega), \omega) d s .
\end{aligned}
$$

If we assume that

$$
\begin{aligned}
x(t, \omega) \stackrel{\left[t_{k-1}, t_{k}\right), \mathbb{P} .1}{=} & x_{0}(\omega)+\int_{t_{0}}^{t} f(s, x(s, \omega), \omega) d s \\
& +\sum_{i=1}^{k-1} I_{i}\left(x\left(t_{i}, \omega\right), \omega\right),
\end{aligned}
$$

then we have

$$
\begin{gathered}
x(t, \omega) \stackrel{\left[t_{k}, t_{k+1}\right], \mathbb{P} .1}{=} x\left(t_{k}^{+}, \omega\right)+\int_{t_{k}}^{t} f(s, x(s, \omega), \omega) d s \\
\stackrel{\left[t_{k}, t_{k+1}\right], \mathbb{P} .1}{=} I_{1}\left(x_{k}(t, \omega), \omega\right)+\int_{t_{k}}^{t} f(s, x(s, \omega), \omega) d s \\
\vdots \\
\stackrel{\left[t_{k}, t_{k+1}\right], \mathbb{P} .1}{=} \sum_{i=1}^{k} I_{i}\left(x\left(t_{i}, \omega\right), \omega\right)+x_{0}(\omega) \\
+\int_{t_{0}}^{t} f(s, x(s, \omega), \omega) d s .
\end{gathered}
$$

It follows by mathematical induction that (13) holds for any $k \geq 1$.

Step 2. Conversely, if a fuzzy stochastic process $x$ satisfies the random fuzzy integral equation (9), then it is equivalent to problem (8). Indeed, if $t \in\left[t_{0}, t_{1}\right)$ we easily see that $x\left(t_{0}, \omega\right) \stackrel{\mathbb{P} .1}{=} x_{0}(\omega)$ and the Hukuhara difference $x_{0}(\omega)+$ $\int_{t_{0}}^{t} f(s, u(s, \omega), \omega) d s$ exists, with P.1. By Lemma 3.2 in [13] we have

$$
D_{H} x(t, \omega) \stackrel{\left[t_{0}, t_{1}\right), \mathbb{P} .1}{=} f(t, x(t, \omega), \omega) .
$$


Let $h>0$ small enough such that $t-h \in\left[t_{1}, t_{2}\right)$ for every $t \in\left[t_{1}, t_{2}\right)$; we have

$$
\begin{aligned}
x(t, \omega) \ominus x(t-h, \omega) \stackrel{\mathbb{P} .1}{=} & \int_{t_{1}}^{t} f(s, x(s, \omega), \omega) d s \\
& \ominus \int_{t_{1}}^{t-h} f(s, x(s, \omega), \omega) d s \\
\stackrel{\mathbb{P} .1}{=} & \int_{t-h}^{t} f(s, x(s, \omega), \omega) d s .
\end{aligned}
$$

Similarly, let $h>0$ small enough such that $t+h \in\left(t_{1}, t_{2}\right)$ for every $t \in\left(t_{1}, t_{2}\right)$; we obtain

$$
\begin{aligned}
& x(t+h, \omega) \ominus x(t, \omega) \stackrel{\mathbb{P} .1}{=} \int_{t_{1}}^{t} f(s, x(s, \omega), \omega) d s \\
& \ominus \int_{t_{1}}^{t+h} f(s, x(s, \omega), \omega) d s \\
& \stackrel{\mathbb{P} .1}{=} \int_{t}^{t+h} f(s, x(s, \omega), \omega) d s .
\end{aligned}
$$

Multiplying both sides of (16) and (17) by $1 / h$ and passing to the limit with $h \rightarrow 0^{+}$, we obtain

$$
\begin{aligned}
& \lim _{h \rightarrow 0^{+}} \frac{x(t, \omega) \ominus x(t-h, \omega)}{h} \\
& =\lim _{h \rightarrow 0^{+}} \frac{1}{h} \int_{t-h}^{t} f(s, x(s, \omega), \omega) d s=D_{H} x(t, \omega), \\
& \lim _{h \rightarrow 0^{+}} \frac{x(t+h, \omega) \ominus x(t, \omega)}{h} \\
& =\lim _{h \rightarrow 0^{+}} \frac{1}{h} \int_{t}^{t+h} f(s, x(s, \omega), \omega) d s=D_{H} x(t, \omega) .
\end{aligned}
$$

This allows us to claim that $x$ is differentiable on $\left(t_{1}, t_{2}\right]$ and consequently

$$
D_{H} x(t, \omega) \stackrel{\left[t_{1}, t_{2}\right), \mathbb{P} .1}{=} f(t, x(t, \omega), \omega) .
$$

By mathematical induction, if $t \in\left(t_{k}, t_{k+1}\right], k=\overline{1, m}$, we get

$$
D_{H} x(t, \omega) \stackrel{\left(t_{k}, t_{k+1}\right], \mathbb{P} .1}{=} f(t, x(t, \omega), \omega) .
$$

Also, we can easily show that

$$
\Delta x\left(t_{k}, \omega\right) \stackrel{\mathbb{P} .1}{=} I_{k}\left(x\left(t_{k}, \omega\right), \omega\right), \quad k=\overline{1, m} .
$$

The proof is complete.

Lemma 8. Let $(\Omega, \mathscr{F}, \mathbb{P})$ be a probability space. Let $A: \Omega \rightarrow$ $\mathbb{R}_{+}, B_{i}: \Omega \rightarrow \mathbb{R}_{+}, i=0,1,2, \ldots$, and stochastic processes $X, Y: J \times \Omega \rightarrow \mathbb{R}$ be such that

(i) $X(\cdot, \omega)$ is nonnegative and continuous with $\mathbb{P} .1$ and $t_{i}$ are the points of discontinuity of the first of $X(\cdot, \omega)$ with P.1, (ii) $Y(\cdot, \omega)$ is locally Lebesgue integrable with $\mathbb{P} .1$

\section{If}

$$
\begin{gathered}
X(t, \omega) \stackrel{J, \mathbb{P} .1}{\leq} A(\omega)+\int_{t_{0}}^{t} X(s, \omega) Y(s, \omega) d s \\
+\sum_{t_{0} \leq t_{i}<t} B_{i}(\omega) X\left(t_{i}, \omega\right),
\end{gathered}
$$

then we have

$$
\begin{aligned}
& X(t, \omega) \\
& \stackrel{J, \mathbb{P} .1}{\leq} A(\omega) \prod_{t_{0} \leq t_{i}<t}\left(1+B_{i}(\omega)\right) \exp \left(\int_{t_{0}}^{t} Y(s, \omega) d s\right) .
\end{aligned}
$$

Now, we show the main results of this paper.

Theorem 9. Let the mapping $f: J \times E^{d} \times \Omega \rightarrow E^{d}$ be continuous with $\mathbb{P} .1$ and $I_{k}: E^{d} \times \Omega \rightarrow E^{d}$. Assume the following conditions hold:

(A1) There exists a nonnegative constant $L_{1}$ such that $D[f(t, \varphi, \omega), f(t, \phi, \omega)] \leq L_{1} D[\varphi, \phi]$, for every $t \in J$ and $\varphi, \phi \in E^{d}$ with $\mathbb{P} .1$.

(A2) There exists a nonnegative constant $L_{2, k}$ such that $D\left[I_{k}(\varphi, \omega), I_{k}(\phi, \omega)\right] \leq L_{2, k} D[\varphi, \phi]$, for $k=0,1,2, \ldots$, $m$, for every $t \in J$ and $\varphi, \phi \in E^{d}$ with $\mathbb{P} .1$.

(A3) There exists a nonnegative constant $M_{1}$ such that $D\left[f\left(t, x_{0}(\omega), \omega\right), \hat{0}\right] \leq M_{1}$, for every $t \in J$ and $x_{0} \in E^{d}$ with $\mathbb{P} .1$.

Then the random fuzzy differential equation with impulses (9) has a unique solution, provided that

$$
\frac{L_{1} p^{n}}{n !}+\sum_{i=1}^{k} L_{2, i}<1, \quad \text { for any } n \in \mathbb{N} \text {. }
$$

Proof. Define a sequence of the functions $x_{n}: J \times \Omega \rightarrow E^{d}$, $n=0,1,2, \ldots$ as follows: for every $\omega \in \Omega$ let us put

$$
\begin{aligned}
x_{0}(t, \omega)= & x_{0}(\omega), \\
x_{n}(t, \omega)= & x_{0}(\omega)+\int_{t_{0}}^{t} f\left(s, x_{n-1}(s, \omega), \omega\right) d s \\
& +\sum_{i=1}^{k} I_{i}\left(x_{n-1}\left(t_{i}, \omega\right), \omega\right) .
\end{aligned}
$$


For every $t \in J$ and $\omega \in \Omega$, we have

$$
\begin{aligned}
d_{\infty}[ & \left.x_{1}(t, \omega), x_{0}(t, \omega)\right] \\
= & d_{\infty}\left[\int_{t_{0}}^{t} f\left(s, x_{0}(s, \omega), \omega\right) d s, \widehat{0}\right] \\
& +d_{\infty}\left[\sum_{i=1}^{k} I_{i}\left(x_{0}\left(t_{i}, \omega\right), \omega\right), \widehat{0}\right] \\
\leq & \int_{t_{0}}^{t} d_{\infty}\left[f\left(s, x_{0}(\omega), \omega\right), \widehat{0}\right] d s \\
& +\sum_{i=1}^{k} d_{\infty}\left[I_{i}\left(x_{0}(\omega), \omega\right), \widehat{0}\right] \\
\leq & M_{1}\left(t-t_{0}\right)+\sum_{i=1}^{k} d_{\infty}\left[I_{i}\left(x_{0}(\omega), \omega\right), \widehat{0}\right] \\
\leq & p M_{1}+\sum_{i=1}^{k} d_{\infty}\left[I_{i}\left(x_{0}(\omega), \omega\right), \widehat{0}\right]:=M_{0}
\end{aligned}
$$

it follows that $d_{\infty}\left[x_{1}(t, \omega), x_{0}(t, \omega)\right] \stackrel{J, \mathbb{P} .1}{\leq} M_{0}$. Furthermore, by assumptions (A1)-(A2) and (25), we can find that

$$
\begin{aligned}
& d_{\infty}\left[x_{n}(t, \omega), x_{n-1}(t, \omega)\right] \\
& \stackrel{J, \mathbb{P} .1}{\leq} \int_{t_{0}}^{t} d_{\infty}\left[f\left(s, x_{n-1}(s, \omega), \omega\right)\right. \\
& \left.f\left(s, x_{n-2}(s, \omega), \omega\right)\right] d s \\
& +\sum_{i=1}^{k} d_{\infty}\left[I_{i}\left(x_{n-1}\left(t_{i}, \omega\right), \omega\right), I_{i}\left(x_{n-2}\left(t_{i}, \omega\right), \omega\right)\right] \\
& \stackrel{J, \mathbb{P} .1}{\leq} L_{1} \int_{t_{0}}^{t} d_{\infty}\left[x_{n-1}(s, \omega), x_{n-2}(s, \omega)\right] d s \\
& +\sum_{i=1}^{k} L_{2, i} d_{\infty}\left[x_{n-1}\left(t_{i}, \omega\right), x_{n-2}\left(t_{i}, \omega\right)\right] \\
& \stackrel{J, \mathbb{P} .1}{\leq} L_{1} \int_{t_{0}}^{t} \sup _{s \in J} d_{\infty}\left[x_{n-1}(s, \omega), x_{n-2}(s, \omega)\right] d s \\
& +\sum_{i=1}^{k} L_{2, i} d_{\infty}\left[x_{n-1}\left(t_{i}, \omega\right), x_{n-2}\left(t_{i}, \omega\right)\right] \stackrel{J, \mathbb{P} .1}{\leq}\left(L_{1}\right. \\
& \left.\cdot \frac{\left(t-t_{0}\right)^{n-1}}{(n-1) !}+\sum_{i=1}^{k} L_{2, i}\right) D\left[x_{n-1}(\omega), x_{n-2}(\omega)\right],
\end{aligned}
$$

which implies that

$$
\begin{gathered}
D\left[x_{n}(t, \omega), x_{n-1}(t, \omega)\right] \\
\stackrel{J, \mathbb{P} .1}{\leq}\left(L_{1} \frac{\left(t-t_{0}\right)^{n-1}}{(n-1) !}+\sum_{i=1}^{k} L_{2, i}\right) \\
\cdot D\left[x_{n-1}(\omega), x_{n-2}(\omega)\right] .
\end{gathered}
$$

Now, we need to prove that for all $t \in J$ with $\mathbb{P} .1$ the following inequality holds: for any $n=1,2, \ldots$,

$$
\begin{aligned}
D & {\left[x_{n}(t, \omega), x_{n-1}(t, \omega)\right] } \\
& \stackrel{J, \mathbb{P} .1}{\leq}\left(L_{1} \frac{\left(t-t_{0}\right)^{n-1}}{(n-1) !}+\sum_{i=1}^{k} L_{2, i}\right) \\
& \cdot D\left[x_{n-1}(\omega), x_{n-2}(\omega)\right] .
\end{aligned}
$$

Indeed, inequality (29) holds for $n=1$. Further, if inequality (29) is true for any $n=m \geq 1$, then using (25) and assumptions (A1)-(A2), we have

$$
\begin{aligned}
& D\left[x_{m+1}(t, \omega), x_{m}(t, \omega)\right] \stackrel{J, \mathbb{P} .1}{\leq} \int_{t_{0}}^{t} \sup _{s \in J} d_{\infty} \\
& \text { - }\left[f\left(s, x_{m}(s, \omega), \omega\right), f\left(s, x_{m-1}(s, \omega), \omega\right)\right] d s \\
& +\sum_{i=1}^{k} d_{\infty}\left[I_{i}\left(x_{m}\left(t_{i}, \omega\right), \omega\right), I_{i}\left(x_{m-1}\left(t_{i}, \omega\right), \omega\right)\right] \\
& \stackrel{J, \mathbb{P} .1}{\leq} L_{1} \int_{t_{0}}^{t} \sup _{s \in J} d_{\infty}\left[x_{m}(s, \omega), x_{m-1}(s, \omega)\right] d s \\
& +\sum_{i=1}^{k} L_{2, i} d_{\infty}\left[x_{m}\left(t_{i}, \omega\right), x_{m-1}\left(t_{i}, \omega\right)\right] \\
& \stackrel{J, \mathbb{P} .1}{\leq} L_{1} \int_{t_{0}}^{t} \sup _{s \in J} d_{\infty}\left[x_{m}(s, \omega), x_{m-1}(s, \omega)\right] d s \\
& +\sum_{i=1}^{k} L_{2, i} d_{\infty}\left[x_{m}\left(t_{i}, \omega\right), x_{m-1}\left(t_{i}, \omega\right)\right] \stackrel{J, \mathbb{P} .1}{\leq}\left(L_{1}\right. \\
& \left.\frac{\left(t-t_{0}\right)^{m}}{m !}+\sum_{i=1}^{k} L_{2, i}\right) D\left[x_{m}(\omega), x_{m-1}(\omega)\right] .
\end{aligned}
$$

Thus, inequality (29) is true for every $t \in J$ with P.1.

Next, we see that $x_{0}(t, \omega)$ does not depend on $t$ and for the right-side continuity of $x_{1}(\cdot, \omega)$, one obtains

$$
\begin{aligned}
& D\left[x_{1}(t+h, \omega), x_{1}(t, \omega)\right] \\
& \quad \stackrel{\left[t_{0}, t_{0}+p\right), \mathbb{P} .1}{\leq} \int_{t}^{t+h} D\left[f\left(s, x_{0}(s, \omega), \omega\right), \widehat{0}\right] d s \\
& \quad+\sum_{i=1}^{k} D\left[I_{i}\left(x_{0}\left(t_{i}+h, \omega\right), \omega\right), I_{i}\left(x_{0}\left(t_{i}, \omega\right), \omega\right)\right] .
\end{aligned}
$$

From the assumption (A3) and $D\left[I_{i}\left(x_{0}\left(t_{i}+h, \omega\right), \omega\right), I_{i}\left(x_{0}\left(t_{i}\right.\right.\right.$, $\omega), \omega)] \rightarrow 0$ as $h \rightarrow 0^{+}$with $\mathbb{P} .1$, we imply that $d_{\infty}\left[x_{1}(t+h, \omega)\right.$, $\left.x_{1}(t, \omega)\right] \rightarrow 0$ as $h \rightarrow 0^{+}$with $\mathbb{P} .1$. 
For every $n \geq 2$, we deduce that

$$
\begin{aligned}
& D\left[x_{n}(t+h, \omega), x_{n}(t, \omega)\right] \\
& \quad \stackrel{\left[t_{0}, t_{0}+p\right), \mathbb{P} .1}{\leq} \int_{t}^{t+h}\left(D\left[f\left(s, x_{0}(s, \omega), \omega\right), \widehat{0}\right]\right. \\
& \left.\quad+\sum_{q=1}^{n-1} D\left[f\left(s, x_{q}(s, \omega), \omega\right), f\left(s, x_{q-1}(s, \omega), \omega\right)\right]\right) d s \\
& +\sum_{i=1}^{k} D\left[I_{i}\left(x_{0}\left(t_{i}+h, \omega\right), \omega\right), I_{i}\left(x_{0}\left(t_{i}, \omega\right), \omega\right)\right] .
\end{aligned}
$$

Using inequality (29) and assumption (A3), we get

$$
\begin{aligned}
D\left[x_{n}(t+h, \omega), x_{n}(t, \omega)\right] \longrightarrow & \\
& \text { as } h \longrightarrow 0^{+} \text {with } \mathbb{P} .1 .
\end{aligned}
$$

Similar for the left-side continuity, we have $d_{\infty}\left[x_{n}(t-\right.$ $\left.h, \omega), x_{n}(t, \omega)\right] \rightarrow 0$ as $h \rightarrow 0^{+}$. Hence the functions $x_{n}(\cdot, \omega)$, $n \geq 2$, are continuous with $\mathbb{P} .1$.

For $n \in \mathbb{N}$ and $t \in J$ the function $x_{n}(t, \cdot)$ defined by (25) is fuzzy random variable. Indeed, $\left[x_{n}(\cdot)\right]^{\alpha}$ is measurable multifunction for every $\alpha \in[0,1]$; it remains to show the same for the mapping $\omega \mapsto\left[\int_{t_{0}}^{t} f\left(s, x_{n-1}(s, \omega), \omega\right) d s+\right.$ $\left.\sum_{i=1}^{k} I_{i}\left(x_{n-1}\left(t_{i}, \omega\right), \omega\right)\right]^{\alpha}$ which is a measurable multifunction with every $\alpha \in[0,1], n \in \mathbb{N}$, and $t \in J$. Let $\alpha \in[0,1]$ be fixed. By virtue of the definition of fuzzy integral and theorem of Nguyen [23] we obtain

$$
\begin{aligned}
& {\left[\int_{t_{0}}^{t} f\left(s, x_{n-1}(s, \omega), \omega\right) d s+\sum_{i=1}^{k} I_{i}\left(x_{n-1}\left(t_{i}, \omega\right), \omega\right)\right]^{\alpha}} \\
& \quad=\int_{t_{0}}^{t} f\left(s,\left[x_{n-1}(s, \omega)\right]^{\alpha}, \omega\right) d s \\
& \quad+\sum_{i=1}^{k} I_{i}\left(\left[x_{n-1}\left(t_{i}, \omega\right)\right]^{\alpha}, \omega\right) .
\end{aligned}
$$

As the integrand is a multifunction continuous in $s$ and measurable in $\omega$, with any $t \in J$, the mapping

$$
\begin{gathered}
\omega \longmapsto \int_{t_{0}}^{t} f\left(s,\left[x_{n-1}(s, \omega)\right]^{\alpha}, \omega\right) d s \\
\quad+\sum_{i=1}^{k} I_{i}\left(\left[x_{n-1}\left(t_{i}, \omega\right)\right]^{\alpha}, \omega\right)
\end{gathered}
$$

is a measurable multifunction for $n \in \mathbb{N}$. Therefore, for every $t \in J$, the sequence $\left\{x_{n}(t, \cdot)\right\}$ is a sequence of fuzzy random variable. Consequently, $\left\{x_{n}(t, \omega)\right\}$ is a sequence of fuzzy stochastic process.

In the sequel, for any $n \in \mathbb{N}$, we shall prove that the sequence $\left\{x_{n}(t, \omega)\right\}$ is a Cauchy sequence uniformly on the variable $t$ with $\mathbb{P} .1$ and then $\left\{x_{n}(\cdot, \omega)\right\}$ is uniformly convergent with P.1.
For any $n \in \mathbb{N}$ and by inequality (29), we obtain

$$
\begin{aligned}
& D\left[x_{n+1}(t, \omega), x_{n}(t, \omega)\right] \stackrel{J, \mathbb{P} .1}{\leq} M D\left[x_{n}(\omega), x_{n-1}(\omega)\right] \\
& \quad \stackrel{J, \mathbb{P} .1}{\leq} M^{n} D\left[x_{1}(\omega), x_{0}(\omega)\right] .
\end{aligned}
$$

Notice now that, for every $m>n>0$, we have

$$
\begin{aligned}
D & {\left[x_{m}(t, \omega), x_{n}(t, \omega)\right] } \\
& \stackrel{J, \mathbb{P} .1}{\leq} \sum_{l=n}^{m-1} D\left[x_{l+1}(t, \omega), x_{l}(t, \omega)\right] \\
& \stackrel{J, \mathbb{P} .1}{\leq}\left(M^{n}+M^{n+1}+\cdots+M^{m-1}\right) D\left[x_{1}(\omega), x_{0}(\omega)\right] \\
& \quad \leq, \mathbb{P} .1 \\
\quad & \frac{M^{n}}{1-M} D\left[x_{1}(\omega), x_{0}(\omega)\right] .
\end{aligned}
$$

For $m>n>0$ large enough, it follows from the above inequalities with $M<1$ that

$$
D\left[x_{m}(t, \omega), x_{n}(t, \omega)\right] \stackrel{\mathbb{P} .1}{\longrightarrow} 0 .
$$

Since $\left(E^{d}, D\right)$ is a complete metric space and (38) holds, then $D\left[x_{n}(t, \omega), x(t, \omega)\right] \stackrel{\mathbb{P} .1}{\longrightarrow} 0$, which means that there exists $\Omega_{0} \subset$ $\Omega$ such that $\mathbb{P}(\Omega)=1$ and for every $\omega \in \Omega_{0}$ the sequence $\left\{x_{n}(\cdot, \omega)\right\}$ is uniformly convergent.

In the following, we shall show that $x(t, \omega)$ is solution of the random impulsive fuzzy integral equation (8). Let $n \in \mathbb{N}$. Observe that

$$
\begin{aligned}
& D\left[\int_{t_{0}}^{t} f\left(s, x_{n-1}(s, \omega), \omega\right) d s, \int_{t_{0}}^{t} f(s, x(s, \omega), \omega) d s\right] \\
& \stackrel{J, \mathbb{P} .1}{\leq} \int_{t_{0}}^{t} D\left[f\left(s, x_{n-1}(s, \omega), \omega\right), f(s, x(s, \omega), \omega)\right] d s \\
& \quad \stackrel{\mathbb{P} .1}{\leq} L_{1} \int_{t_{0}}^{t} D\left[x_{n-1}(s, \omega), x(s, \omega)\right] d s .
\end{aligned}
$$

Since the sequence $x_{n}(t, \omega)$ converges uniformly to $x(t, \omega)$ on the variable $t \in J$ with $\mathbb{P} .1$ as $n \rightarrow+\infty$, Thus for any $\varepsilon>0$ there is $n_{0}>0$ large enough such that, for all $n>n_{0}$, we derive

$$
\begin{aligned}
& D\left[x_{n-1}(t, \omega), x(t, \omega)\right] \\
& \quad \stackrel{J, \mathbb{P} .1}{\leq} \min \left\{\frac{(n-1) !}{L_{1} p^{n-1}} \varepsilon,\left(\sum_{i=1}^{k} L_{2, i}\right)^{-1} \varepsilon\right\} .
\end{aligned}
$$

Therefore,

$$
\begin{aligned}
& D\left[\int_{t_{0}}^{t} f\left(s, x_{n-1}(s, \omega), \omega\right) d s, \int_{t_{0}}^{t} f(s, x(s, \omega), \omega) d s\right] \\
& J, \mathbb{P} .1 \\
& \leq \varepsilon \text {, } \\
& D\left[\sum_{i=1}^{k} I_{i}\left(x_{n-1}\left(t_{i}, \omega\right), \omega\right), \sum_{i=1}^{k} I_{i}\left(x\left(t_{i}, \omega\right), \omega\right)\right] \\
& \stackrel{J, \mathbb{P} .1}{\leq} \sum_{i=1}^{k} L_{2, i} D\left[x_{n-1}\left(t_{i}, \omega\right), x\left(t_{i}, \omega\right)\right] \stackrel{J, \mathbb{P} .1}{\leq} \varepsilon .
\end{aligned}
$$


On the other hand, we have

$$
\begin{aligned}
& D\left[x(t, \omega), x_{0}(\omega)+\int_{t_{0}}^{t} f(s, x(s, \omega), \omega) d s\right. \\
& \left.+\sum_{i=1}^{k} I_{i}\left(x\left(t_{i}, \omega\right), \omega\right)\right] \stackrel{j, \mathbb{P} .1}{\leq} D\left[x(t, \omega), x_{n}(t, \omega)\right] \\
& +d_{\infty}\left[x_{n}(t, \omega), x_{0}(\omega)\right. \\
& +\int_{t_{0}}^{t} f\left(s, x_{n-1}(s, \omega), \omega\right) d s \\
& \left.+\sum_{i=1}^{k} I_{i}\left(x_{n-1}\left(t_{i}, \omega\right), \omega\right)\right] \\
& \quad+D\left[\int_{t_{0}}^{t} f\left(s, x_{n-1}(s, \omega), \omega\right) d s\right. \\
& \left.\quad \int_{t_{0}}^{t} f(s, x(s, \omega), \omega) d s\right]+D\left[\sum_{i=1}^{k} I_{i}\left(x_{n-1}\left(t_{i}, \omega\right), \omega\right)\right. \\
& \left.\sum_{i=1}^{k} I_{i}\left(x\left(t_{i}, \omega\right), \omega\right)\right]
\end{aligned}
$$

Thus, in view of the convergence of the two previous equations and (41), one obtains that

$$
\begin{gathered}
D\left[x(t, \omega), x_{0}(\omega)+\int_{t_{0}}^{t} f(s, x(s, \omega), \omega) d s\right. \\
\left.\quad+\sum_{i=1}^{k} I_{i}\left(x\left(t_{i}, \omega\right), \omega\right)\right] \stackrel{J, \mathbb{P} .1}{=} 0 .
\end{gathered}
$$

It means the fuzzy stochastic process $x(t, \omega)$ is solution of problem (8).

To prove the uniqueness, let us assume that $x, y: J \times \Omega \rightarrow$ $E^{d}$ are the two continuous fuzzy stochastic processes which are solutions of problem (8). Note that

$$
\begin{aligned}
& D[x(t, \omega), y(t, \omega)] \stackrel{J, \mathbb{P} .1}{=} D\left[\int_{t_{0}}^{t} f(s, x(s, \omega), \omega) d s,\right. \\
& \left.\int_{t_{0}}^{t} f(s, y(s, \omega), \omega) d s\right]+D\left[\sum_{i=1}^{k} I_{i}\left(x\left(t_{i}, \omega\right), \omega\right),\right. \\
& \left.\sum_{i=1}^{k} I_{i}\left(y\left(t_{i}, \omega\right), \omega\right)\right] \stackrel{\text { J,P.1 }}{\leq}\left(\frac{L_{1} p^{n}}{n !}+\sum_{i=1}^{k} L_{2, i}\right) D[x(\omega), \\
& y(\omega)] .
\end{aligned}
$$

By Lemma 8, we get

$$
D[x(t, \omega), y(t, \omega)] \stackrel{J, \mathbb{P} .1}{\leq} 0 .
$$

The uniqueness is proved. The proof is complete.

\section{Some of the Properties of Solution of RFDEs with Impulses}

Theorem 10. Suppose that the mappings $f: J \times E^{d} \times \Omega \rightarrow E^{d}$ and $I_{k}: E^{d} \times \Omega \rightarrow E^{d}$ satisfy all the conditions of Theorem 9 . Then we have

$$
\begin{aligned}
& D[x(t, \omega), \hat{0}] \stackrel{J, \mathbb{P} .1}{\leq}\left(D\left[x_{0}(\omega), \hat{0}\right]+\left(t-t_{0}\right) M_{1}\right) \\
& \cdot \prod_{t_{0} \leq t_{i}<t}\left(1+L_{2, i}\right) \\
& \cdot \exp \left(L_{1}\left(t-t_{0}\right)\right)
\end{aligned}
$$

where $L_{1}, L_{2, i}$ are constants nonnegative for any $i=$ $0,1,2,3, \ldots$

Proof. Let $x(t, \omega)$ be solution of problem (8). For every $t \in$ $\left[t_{0}, t_{1}\right)$ and $\omega \in \Omega$, we have

$$
\begin{aligned}
D[ & x(t, \omega), \widehat{0}] \\
\leq & D\left[x_{0}(\omega), \widehat{0}\right]+D\left[\int_{t_{0}}^{t} f(s, x(s, \omega), \omega) d s, \widehat{0}\right] \\
\leq & D\left[x_{0}(\omega), \hat{0}\right]+\int_{t_{0}}^{t} D[f(s, x(s, \omega), \omega), \widehat{0}] d s \\
\leq & \mathrm{D}\left[x_{0}(\omega), \hat{0}\right]+\int_{t_{0}}^{t} D[f(s, \widehat{0}, \omega), \widehat{0}] \\
& +D[f(s, x(s, \omega), \omega), f(s, \widehat{0}, \omega)]) d s \\
\leq & D\left[x_{0}(\omega), \hat{0}\right]+\left(t-t_{0}\right) D[f(s, \widehat{0}, \omega), \widehat{0}] \\
& +L_{1} \int_{t_{0}}^{t} D[x(s, \omega), \widehat{0}] d s \\
\leq & D\left[x_{0}(\omega), \widehat{0}\right]+\left(t-t_{0}\right) M_{1} \\
& +\int_{t_{0}}^{t} L_{1} D[x(s, \omega), \widehat{0}] d s .
\end{aligned}
$$

For every $t \in\left[t_{k}, t_{k+1}\right), k=1,2,3, \ldots$, and $\omega \in \Omega$, we have

$$
\begin{aligned}
& D[x(t, \omega), \widehat{0}] \leq d_{\infty}\left[x_{0}(\omega), \widehat{0}\right] \\
& +D\left[\int_{t_{0}}^{t} f(s, x(s, \omega), \omega) d s, \widehat{0}\right] \\
& +D\left[\sum_{i=1}^{k} I_{i}\left(x\left(t_{i}, \omega\right), \omega\right), \widehat{0}\right] \leq D\left[x_{0}(\omega), \widehat{0}\right] \\
& +\int_{t_{0}}^{t}(D[f(s, \widehat{0}, \omega), \widehat{0}] \\
& +D[f(s, x(s, \omega), \omega), f(s, \widehat{0}, \omega)]) d s
\end{aligned}
$$




$$
\begin{aligned}
& +\sum_{i=1}^{k} D\left[I_{i}\left(x\left(t_{i}, \omega\right), \omega\right), \widehat{0}\right] \leq D\left[x_{0}(\omega), \widehat{0}\right]+(t \\
& \left.-t_{0}\right) M_{1}+\int_{t_{0}}^{t} L_{1} D[x(s, \omega), \widehat{0}] d s \\
& +\sum_{i=1}^{k} L_{2, k} D\left[x\left(t_{i}, \omega\right), \widehat{0}\right] .
\end{aligned}
$$

If we let $\xi(t, \omega)=D[x(t, \omega), \widehat{0}], t \in\left[t_{k}, t_{k+1}\right)$, and $k=0,1,2$, $3, \ldots$, then we have

$$
\begin{gathered}
\xi(t, \omega) \stackrel{\mathbb{P} .1}{\leq} \xi_{0}(\omega)+\left(t-t_{0}\right) M_{1}+\int_{t_{0}}^{t} L_{1} \xi(s, \omega) d s \\
+\sum_{i=1}^{k} L_{2, i} \xi\left(t_{i}, \omega\right) .
\end{gathered}
$$

By virtue of Lemma 8, one obtains

$$
\begin{aligned}
\xi(t, \omega) \stackrel{\mathbb{P} .1}{\leq} & \left(\xi_{0}(\omega)+\left(t-t_{0}\right) M_{1}\right) \prod_{t_{0} \leq t_{i}<t}\left(1+L_{2, i}\right) \\
& \cdot \exp \left(L_{1}\left(t-t_{0}\right)\right) .
\end{aligned}
$$

The proof is complete.

Theorem 11. Suppose that the mappings $f: J \times E^{d} \times \Omega \rightarrow E^{d}$ and $I_{k}: E^{d} \times \Omega \rightarrow E^{d}$ satisfy all the conditions of Theorem 9 . Then we have

$$
\begin{gathered}
D[x(t, \omega), y(t, \omega)] \stackrel{J, \mathbb{P} .1}{\leq} D\left[x_{0}(\omega), y_{0}(\omega)\right] \\
\cdot \prod_{t_{0} \leq t_{i}<t}\left(1+L_{2, i}\right) \exp \left(L_{1}\left(t-t_{0}\right)\right),
\end{gathered}
$$

where $L_{1}, L_{2, i}$ are constants nonnegative for any $i=$ $0,1,2,3, \ldots$.

Proof. Let $x(t, \omega)$ and $y(t, \omega)$ be solutions of problem (8). For every $t \in\left[t_{0}, t_{1}\right)$ and $\omega \in \Omega$, we have

$$
\begin{aligned}
& D[x(t, \omega), y(t, \omega)] \leq d_{\infty}\left[x_{0}(\omega), y_{0}(\omega)\right] \\
& +D\left[\int_{t_{0}}^{t} f(s, x(s, \omega), \omega) d s\right. \\
& \left.\quad \int_{t_{0}}^{t} f(s, y(s, \omega), \omega) d s\right] \leq D\left[x_{0}(\omega), y_{0}(\omega)\right] \\
& +\int_{t_{0}}^{t} D[f(s, x(s, \omega), \omega), f(s, y(s, \omega), \omega)] d s \\
& \quad=D\left[x_{0}(\omega), y_{0}(\omega)\right] \\
& +L_{1} \int_{t_{0}}^{t} D[x(s, \omega), y(s, \omega)] d s .
\end{aligned}
$$

For every $t \in\left[t_{k}, t_{k+1}\right), k=1,2,3, \ldots$, and $\omega \in \Omega$, we have

$$
\begin{aligned}
& D[x(t, \omega), y(t, \omega)] \leq d_{\infty}\left[x_{0}(\omega), y_{0}(\omega)\right] \\
& +D\left[\int_{t_{0}}^{t} f(s, x(s, \omega), \omega) d s\right. \\
& \left.\quad \int_{t_{0}}^{t} f(s, y(s, \omega), \omega) d s\right]+D\left[\sum_{i=1}^{k} I_{i}\left(x\left(t_{i}, \omega\right), \omega\right),\right. \\
& \left.\quad \sum_{i=1}^{k} I_{i}\left(y\left(t_{i}, \omega\right), \omega\right)\right] \leq D\left[x_{0}(\omega), y_{0}(\omega)\right] \\
& +\int_{t_{0}}^{t} D[f(s, x(s, \omega), \omega), f(s, y(s, \omega), \omega)] d s \\
& +\sum_{i=1}^{k} d_{\infty}\left[I_{i}\left(x\left(t_{i}, \omega\right), \omega\right), I_{i}\left(y\left(t_{i}, \omega\right), \omega\right)\right] \\
& \quad=D^{t}\left[x_{0}(\omega), y_{0}(\omega)\right] \\
& +\int_{t_{0}}^{t} L_{1} D[x(s, \omega), y(s, \omega)] d s \\
& +\sum_{i=1}^{k} L_{2, i} D\left[x\left(t_{i}, \omega\right), y\left(t_{i}, \omega\right)\right] .
\end{aligned}
$$

If we let $\xi(t, \omega)=D\left[x_{0}(\omega), y_{0}(\omega)\right], t \in\left[t_{k}, t_{k+1}\right)$, and $k=$ $0,1,2,3, \ldots$, then we have

$$
\xi(t, \omega) \stackrel{\mathbb{P} .1}{\leq} \xi_{0}(\omega)+\int_{t_{0}}^{t} L_{1} \xi(s, \omega) d s+\sum_{i=1}^{k} L_{2, i} \xi\left(t_{i}, \omega\right)
$$

By virtue of Lemma 8, one obtains

$$
\xi(t, \omega) \stackrel{\mathbb{P} .1}{\leq} \xi_{0}(\omega) \prod_{t_{0} \leq t_{i}<t}\left(1+L_{2, i}\right) \exp \left(L_{1}\left(t-t_{0}\right)\right)
$$

The proof is complete.

\section{Illustrative Examples}

In this section, we shall consider two examples. First, we give an example to illustrate the existence and uniqueness results obtained in Section 3. Second, we will find explicit representation of solutions RFDEs with impulses.

Example 1. Let $\Omega=(0,1), \mathscr{F}$-Borel $\sigma$-algebra of subsets of $\Omega$, and $\mathbb{P}$-Lebesgue measure on $(\Omega, \mathscr{F})$. Let us consider the problem as follows:

$$
D_{H} x(t, \omega) \stackrel{[0,1], \mathbb{P} .1}{=} \frac{\exp (-t)}{(5+\exp (t))(1+x(t, \omega))},
$$




$$
\begin{aligned}
& x\left(t_{k}^{+}, \omega\right) \stackrel{\mathbb{P} .1}{=} \frac{x\left(t_{k}, \omega\right)}{2+x\left(t_{k}, \omega\right)}, \\
& \qquad t=t_{k}, k=0,1,2, \ldots, m, \\
& x(0, \omega) \stackrel{\mathbb{P} .1}{=}(-1,0,1,2) \omega \in E^{1},
\end{aligned}
$$

where $x:[0,1] \times \Omega \rightarrow E^{1}$ is a fuzzy stochastic process. Set

$$
\begin{aligned}
& f(t, x(t, \omega), \omega)=\frac{\exp (-t)}{(5+\exp (t))(1+x(t, \omega))}, \\
& \quad \text { for every } t \in[0,1], t \neq t_{k}, k=0,1,2, \ldots, m, \\
& I_{k}\left(x_{k}(t, \omega), \omega\right)=\frac{x\left(t_{k}, \omega\right)}{2+x\left(t_{k}, \omega\right)}, \\
& \quad \text { for every } t \in[0,1], t=t_{k}, k=0,1,2, \ldots, m .
\end{aligned}
$$

For every $t \in[0,1], t \neq t_{k}, k=0,1,2, \ldots, m$, we have

$$
\begin{aligned}
& d_{\infty}[f(t, x(t, \omega), \omega), f(t, y(t, \omega), \omega)] \\
& =d_{\infty}\left[\frac{\exp (-t)}{(5+\exp (t))(1+x(t, \omega))},\right. \\
& \left.\frac{\exp (-t)}{(5+\exp (t))(1+y(t, \omega))}\right] \stackrel{[0,1], \mathbb{P} .1}{=} \frac{\exp (-t)}{5+\exp (t)} \\
& \cdot d_{\infty}\left[\frac{1}{1+x(t, \omega)}, \frac{1}{1+y(t, \omega)}\right] \\
& \stackrel{[0,1], \mathbb{P} .1}{=} \frac{\exp (-t)}{5+\exp (t)} \\
& \cdot \sup _{\alpha \in[0,1]} \max \left\{\left|\frac{1}{1+x_{l \alpha}(t, \omega)}-\frac{1}{1+y_{l \alpha}(t, \omega)}\right|\right. \text {, } \\
& \left.\left|\frac{1}{1+x_{r \alpha}(t, \omega)}-\frac{1}{1+y_{r \alpha}(t, \omega)}\right|\right\} \\
& \stackrel{[0,1], \mathbb{P} .1}{=} \frac{\exp (-t)}{5+\exp (t)} \\
& \cdot \sup _{\alpha \in[0,1]} \max \left\{\left|\frac{x_{l \alpha}(t, \omega)-y_{l \alpha}(t, \omega)}{\left(1+x_{l \alpha}(t, \omega)\right)\left(1+y_{l \alpha}(t, \omega)\right)}\right|\right. \text {, } \\
& \left.\left|\frac{x_{r \alpha}(t, \omega)-y_{r \alpha}(t, \omega)}{\left(1+x_{r \alpha}(t, \omega)\right)\left(1+y_{r \alpha}(t, \omega)\right)}\right|\right\} \stackrel{[0,1], \mathbb{P} .1}{\leq} \frac{1}{6} \\
& \text { - } \sup _{\alpha \in[0,1]} \max \left\{\left|x_{l \alpha}(t, \omega)-y_{l \alpha}(t, \omega)\right|\right. \text {, } \\
& \left.\left|x_{r \alpha}(t, \omega)-y_{r \alpha}(t, \omega)\right|\right\} \stackrel{[0,1], \mathbb{P} .1}{\leq} \frac{1}{6} d_{\infty}[x, y],
\end{aligned}
$$

where $L_{1}=\sup _{t \in[0,1]}(\exp (-t) /(5+\exp (t)))=1 / 6$.
Using a similar calculation as above, for every $t \in[0,1]$, $t=t_{k}, k=0,1,2, \ldots, m$, we obtain

$$
\begin{aligned}
& d_{\infty}\left[I_{k}\left(x_{k}(t, \omega), \omega\right), I_{k}\left(y_{k}(t, \omega), \omega\right)\right] \\
& \stackrel{[0,1], \mathbb{P} .1}{\leq} \frac{1}{2} d_{\infty}[x, y], \quad \text { where } L_{2, k}=\frac{1}{2} .
\end{aligned}
$$

By a direct calculation, one obtains that

$$
\begin{aligned}
& d_{\infty}\left[\frac{\exp (-t)}{(5+\exp (t))(1+x(t, \omega))}, \widehat{0}\right] \\
& \stackrel{[0,1], \mathbb{P} .1}{=} \frac{\exp (-t)}{5+\exp (t)} d_{\infty}\left[\frac{1}{1+x(t, \omega)}, \widehat{0}\right] \\
& \stackrel{[0,1], \mathbb{P} .1}{=} \frac{\exp (-t)}{5+\exp (t)} \\
& \cdot \sup _{\alpha \in[0,1]}^{\max }\left\{\left|\frac{1}{1+x_{l \alpha}(t, \omega)}\right|,\left|\frac{1}{1+x_{r \alpha}(t, \omega)}\right|\right\} \\
& \stackrel{[0,1], \mathbb{P} .1}{\leq} \frac{1}{6}
\end{aligned}
$$

and for any $n=1,2, \ldots$,

$$
\frac{L_{1} p^{n}}{n !}+\sum_{i=1}^{k} L_{2, i}=\frac{1}{6^{n} n !}+\frac{1}{2}<1 .
$$

We can see that conditions (A1)-(A4) are satisfied. Hence, by Theorem 9, problem (56) has a solution defined on $[0,1]$.

Example 2. Let $\Omega=(0,1), \mathscr{F}$-Borel $\sigma$-algebra of subsets of $\Omega$, and $\mathbb{P}$-Lebesgue measure on $(\Omega, \mathscr{F})$. Consider the RFDEs with impulses as follows:

$$
\begin{aligned}
& D_{H} x(t, \omega) \stackrel{[0, T], \mathbb{P} .1}{=} \lambda(\omega) x(t, \omega), \\
& t \neq t_{k}, k=0,1,2, \ldots, m, \\
& x\left(t_{k}^{+}, \omega\right) \stackrel{\mathbb{P} .1}{=} x_{k}\left(t_{k}, \omega\right)+I_{k}\left(x_{k}(t, \omega), \omega\right), \\
& \quad t=t_{k}, k=0,1,2, \ldots, m, \\
& x(0, \omega) \stackrel{\mathbb{P} .1}{=} x_{0}(\omega),
\end{aligned}
$$

where $\lambda: \Omega \rightarrow \mathbb{R}_{+}$is a random variable and $x:[0,1] \times \Omega \rightarrow$ $E^{1}$ is a fuzzy stochastic process. In this example, we suppose that $t \in[0,2]$ and $\lambda(\omega)=1$ with $\mathbb{P} .1$ and for every $\alpha \in[0,1]$

$$
\begin{aligned}
{[x(t, \omega)]^{\alpha} } & =\left[x_{l \alpha}(t, \omega), x_{r \alpha}(t, \omega)\right] \\
{\left[x_{k}\left(t_{k}, \omega\right)\right]^{\alpha} } & =[(\omega, 2 \omega, 3 \omega)]^{\alpha} \\
& =[(1+\alpha) \omega,(3-\alpha) \omega], \\
& t=k, k=1,2,
\end{aligned}
$$

and initial conditions $\left[x_{0}(\omega)\right]^{\alpha}=[(-\omega, 0, \omega)]^{\alpha}=[(\alpha-$ 1) $\omega,(1-\alpha) \omega]$, where $x_{l \alpha}, x_{r \alpha}:[0, \infty) \times \Omega \rightarrow \mathbb{R}$ are the crisp stochastic process. 
Problem (62) can translate this into the following system of random differential equation with impulses:

$$
\begin{aligned}
x_{l \alpha}^{\prime}(t, \omega) & =x_{l \alpha}(t, \omega), \quad t \in[0,2], t \neq t_{k}, \\
x_{r \alpha}^{\prime}(t, \omega) & =x_{r \alpha}(t, \omega), \quad t \in[0,2], t \neq t_{k}, \\
x_{l \alpha}\left(t_{k}^{+}, \omega\right) & =(1+\alpha) \omega, \quad t=k, k=1,2, \\
x_{r \alpha}\left(t_{k}^{+}, \omega\right) & =(3-\alpha) \omega, \quad t=k, k=1,2, \\
x_{l \alpha}(0, \omega) & =(\alpha-1) \omega, \\
x_{r \alpha}(0, \omega) & =(1-\alpha) \omega .
\end{aligned}
$$

Solving system $(64)$ on $[0,2]$, we obtain

$$
\begin{aligned}
& x_{l \alpha}(t, \omega)= \begin{cases}(\alpha-1) \omega \exp (t), & \text { for } t \in[0,1), \\
(1+\alpha) \omega \exp (t-1), & \text { for } t \in[0,2),\end{cases} \\
& x_{r \alpha}(t, \omega)= \begin{cases}(1-\alpha) \omega \exp (t), & \text { for } t \in[0,1), \\
(3-\alpha) \omega \exp (t-1), & \text { for } t \in[0,2) .\end{cases}
\end{aligned}
$$

It is easy to see that the diameter of solution $x(t, \omega)$ of $(62)$ is an increasing function with $\mathbb{P} .1$ for every $t \in[0,2]$. Hence we infer that the solution $x:[0,2] \times \Omega \rightarrow E^{1}$ to (62) is as follows:

$$
\begin{aligned}
& x(t, \omega) \\
& = \begin{cases}{[(\alpha-1),(1-\alpha)] \omega \exp (t),} & \text { for } t \in[0,1), \\
{[(1+\alpha),(3-\alpha)] \omega \exp (t-1),} & \text { for } t \in[0,2)\end{cases}
\end{aligned}
$$

or

$$
x(t, \omega)= \begin{cases}(-1,0,1) \omega \exp (t), & \text { for } t \in[0,1), \\ (1,2,3) \omega \exp (t-1), & \text { for } t \in[0,2) .\end{cases}
$$

Note that the existence of a unique solution is guaranteed. Therefore, this procedure can be continued to be the solution on each $[m, m+1]$, for every $m \in \mathbb{N}, m \geq 3$.

\section{Conclusion}

Under suitable conditions, we investigated the existence and uniqueness of solutions to random fuzzy differential equation with impulses by using the method of successive approximations. Moreover, we studied some of the properties of solution of RFDEs with impulses. Finally, some examples are given to illustrate the main theorems. In the future, we shall study the class of random fuzzy differential equations in the quotient space of fuzzy numbers, introduced by Qiu et al. in [24].

\section{Conflicts of Interest}

The author declares that there are no conflicts of interest regarding the publication of this paper.

\section{References}

[1] M. Benchohra, J. Henderson, and S. Ntouyas, Impulsive differential equations and inclusions, vol. 2 of Contemporary Mathematics and Its Applications, Hindawi Publishing Corporation, New York, 2006.

[2] V. Lakshmikantham, D. D. Bainov, and P. S. Simeonov, Theory of impulsive differential equations, vol. 6 of Series in Modern Applied Mathematics, World Scientific Publishing Co., Inc., Teaneck, NJ, 1989.

[3] J. J. Nieto and R. Rodríguez-López, "Periodic boundary value problem for non-Lipschitzian impulsive functional differential equations," Journal of Mathematical Analysis and Applications, vol. 318, no. 2, pp. 593-610, 2006.

[4] A. M. Samoilenko and N. A. Perestyuk, "Impulsive differential equations," World Scientific, 1995.

[5] B. Bede and S. G. Gal, "Generalizations of the differentiability of fuzzy-number-valued functions with applications to fuzzy differential equations," Fuzzy Sets and Systems. An International Journal in Information Science and Engineering, vol. 151, no. 3, pp. 581-599, 2005.

[6] Y. Chalco-Cano and H. Román-Flores, "On new solutions of fuzzy differential equations," Chaos, Solitons and Fractals, vol. 38, no. 1, pp. 112-119, 2008.

[7] O. Kaleva, "Fuzzy differential equations," Fuzzy Sets and Systems. An International Journal in Information Science and Engineering, vol. 24, no. 3, pp. 301-317, 1987.

[8] O. Kaleva, "A note on fuzzy differential equations," Nonlinear Analysis, Theory, Methods and Applications, vol. 64, no. 5, pp. 895-900, 2006.

[9] T. G. Bhaskar, V. Lakshmikantham, and J. V. Devi, "Nonlinear variation of parameters formula for set differential equations in a metric space," Nonlinear Analysis, Theory, Methods and Applications, vol. 63, no. 5-7, pp. 735-744, 2005.

[10] V. Lakshmikantham and F. A. McRae, "Basic results for fuzzy impulsive differential equations," Mathematical Inequalities \& Applications, vol. 4, no. 2, pp. 239-246, 2001.

[11] J. J. Nieto and R. Rodríguez-López, "Some results on boundary value problems for fuzzy differential equations with functional dependence," Fuzzy Sets and Systems, vol. 230, pp. 92-118, 2013.

[12] R. Rodrguez-López, "Periodic boundary value problems for impulsive fuzzy differential equations," Fuzzy Sets and Systems, vol. 159, no. 11, pp. 1384-1409, 2008.

[13] M. T. Malinowski, "On random fuzzy differential equations," Fuzzy Sets and Systems. An International Journal in Information Science and Engineering, vol. 160, no. 21, pp. 3152-3165, 2009.

[14] M. T. Malinowski, "Existence theorems for solutions to random fuzzy differential equations," Nonlinear Analysis, Theory, Methods and Applications, vol. 73, no. 6, pp. 1515-1532, 2010.

[15] M. T. Malinowski, "Random fuzzy differential equations under generalized Lipschitz condition," Nonlinear Analysis. Real World Applications. An International Multidisciplinary Journal, vol. 13, no. 2, pp. 860-881, 2012.

[16] M. T. Malinowski, "Random fuzzy fractional integral equations-theoretical foundations," Fuzzy Sets and Systems. An International Journal in Information Science and Engineering, vol. 265, pp. 39-62, 2015.

[17] Y. Feng, "Mean-square integral and differential of fuzzy stochastic processes," Fuzzy Sets and Systems. An International Journal in Information Science and Engineering, vol. 102, no. 2, pp. 271280, 1999. 
[18] Y. Feng, "Fuzzy stochastic differential systems," Fuzzy Sets and Systems. An International Journal in Information Science and Engineering, vol. 115, no. 3, pp. 351-363, 2000.

[19] W. Fei, "Existence and uniqueness of solution for fuzzy random differential equations with non-Lipschitz coefficients," Information Sciences. An International Journal, vol. 177, no. 20, pp. 43294337, 2007.

[20] W. Fei, "A generalization of Bihari's inequality and fuzzy random differential equations with non-Lipschitz coefficients," International Journal of Uncertainty, Fuzziness and KnowledgeBased Systems, vol. 15, no. 4, pp. 425-439, 2007.

[21] M. Guo, X. Xue, and R. Li, "Impulsive functional differential inclusions and fuzzy population models," Fuzzy Sets and Systems. An International Journal in Information Science and Engineering, vol. 138, no. 3, pp. 601-615, 2003.

[22] V. Lakshmikantham and R. N. Mohapatra, Theory of Fuzzy Differential Equations and Inclusions, vol. 6, Taylor and Francis Publishers, London, UK, 2003.

[23] H. T. Nguyen, "A note on the extension principle for fuzzy sets," Journal of Mathematical Analysis and Applications, vol. 64, no. 2, pp. 369-380, 1978.

[24] D. Qiu, W. Zhang, and C. Lu, "On fuzzy differential equations in the quotient space of fuzzy numbers," Fuzzy Sets and Systems. An International Journal in Information Science and Engineering, vol. 295, pp. 72-98, 2016. 


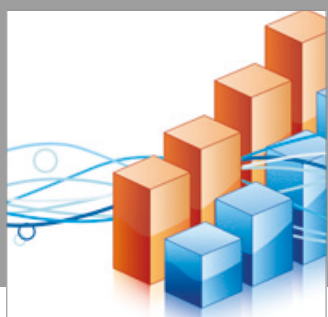

Advances in

Operations Research

vatersals

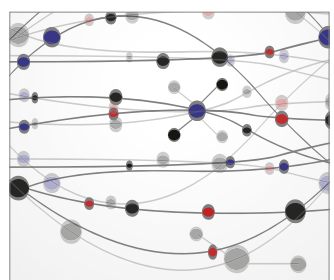

\section{The Scientific} World Journal
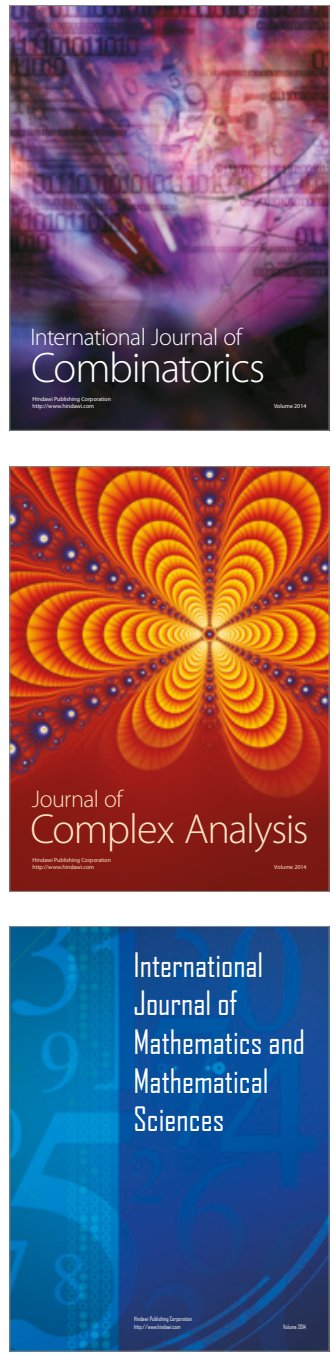
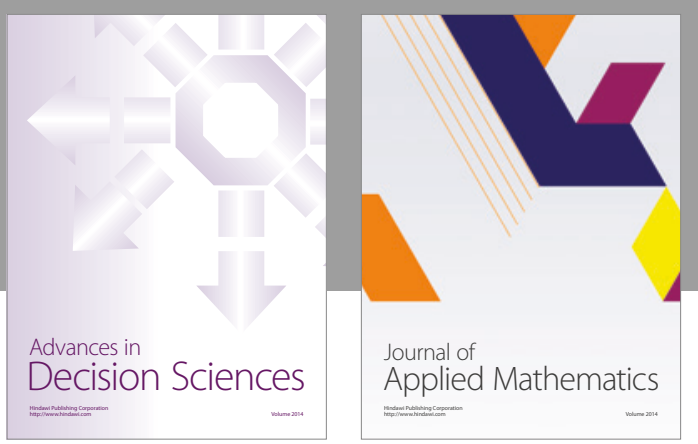

Algebra

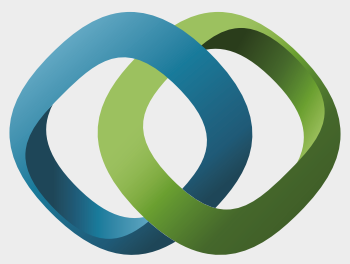

\section{Hindawi}

Submit your manuscripts at

https://www.hindawi.com
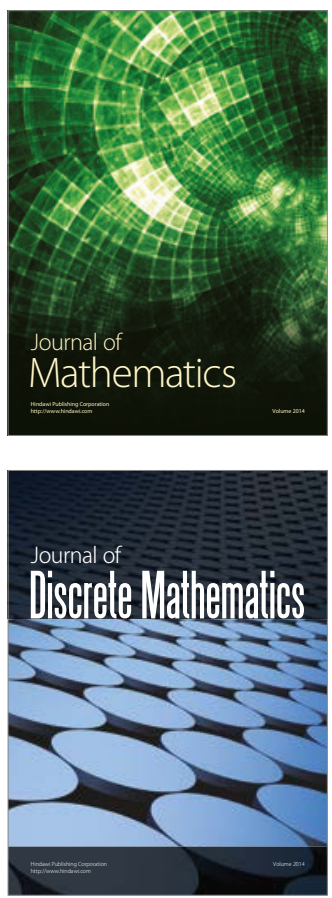

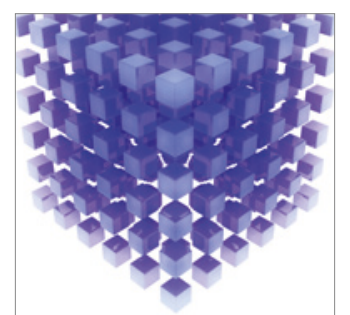

Mathematical Problems in Engineering
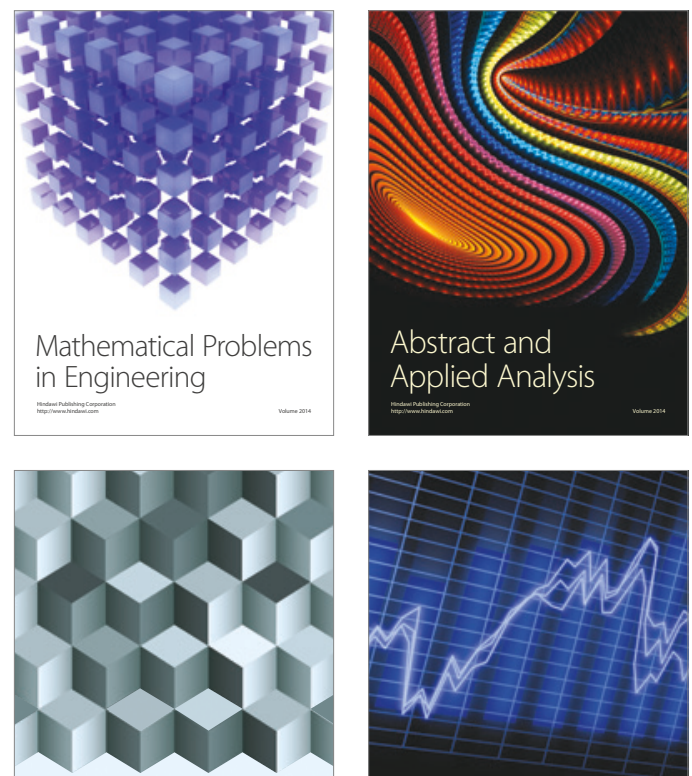

Journal of

Function Spaces

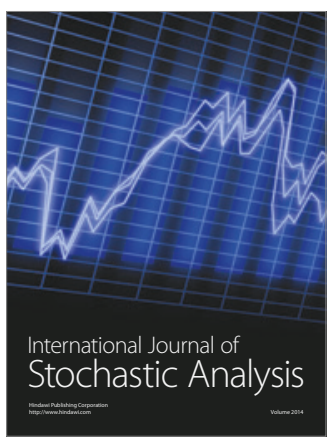

Probability and Statistics
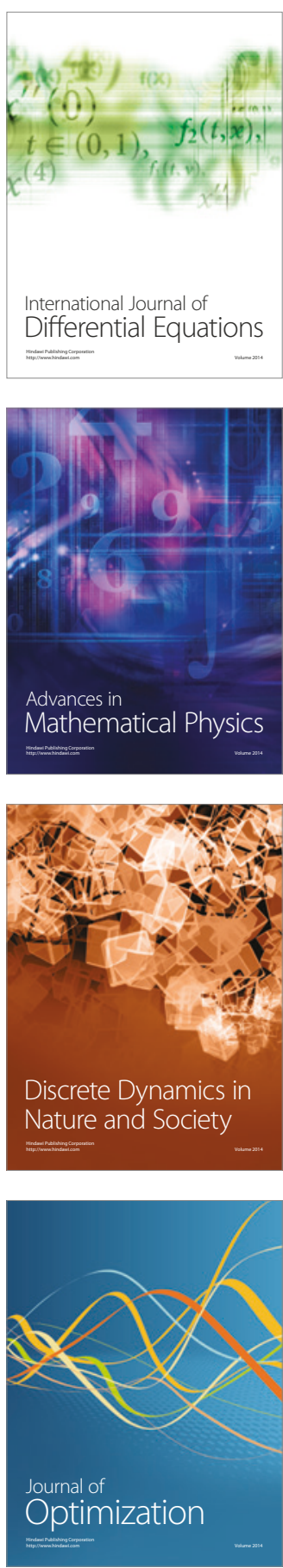